\title{
Where have all the susceptible gonococci gone? A historical review of changes in MIC distribution over the past 75 years
}

\author{
Chris Kenyon ${ }^{1,2^{*}} \mathbb{D}$, Jolein Laumen ${ }^{1}$, Dorien Van Den Bossche ${ }^{1}$ and Christophe Van Dijck
}

\begin{abstract}
Background: Does the emergence of antimicrobial resistance in Neisseria gonorrhoeae include the erasure of highly susceptible strains or does it merely involve a stretching of the MIC distribution? If it was the former this would be important to know as it would increase the probability that the loss of susceptibility is irreversible.

Methods: We conducted a historical analysis based on a literature review of changes of N. gonorrhoeae MIC distribution over the past 75 years for 3 antimicrobials (benzylpenicillin, ceftriaxone and azithromycin) in five countries (Denmark, Japan, South Africa, the United Kingdom and the United States).

Results: Changes in MIC distribution were most marked for benzylpenicillin and showed evidence of a right shifting of MIC distribution that was associated with a reduction/elimination of susceptible strains in all countries. In the case of ceftriaxone and azithromycin, where only more recent data was available, right shifting was also found in all countries but the extent of right shifting varied and the evidence for the elimination of susceptible strains was more mixed.

Conclusions: The finding of right shifting of MIC distribution combined with reduction/elimination of susceptible strains is of concern since it suggests that this shifting may not be reversible. Since excess antimicrobial consumption is likely to be responsible for this right shifting, this insight provides additional impetus to promote antimicrobial stewardship.
\end{abstract}

Keywords: Neisseria gonorrhoeae, Gonococcus, Antimicrobial resistance, AMR, MIC, MIC-shift, ECOFF

\section{Background}

Typically, investigations of antimicrobial resistance (AMR) in Neisseria gonorrhoeae have focused on the right-hand tail of the MIC distributions (the subpopulation of $N$. gonorrhoeae that acquires AMR) [1-3]. An under investigated topic is what happens to the left-hand tail of the MIC distribution and whether or not the emergence of AMR is associated with a right shifting of the entire MIC distribution including the left-hand tail [4-7]? One previous ecological analysis of 5 year data from 24 European countries found that the emergence of gonococcal AMR was associated with a right shift of the susceptible isolates [6]. The study did not however characterize the nature of

\footnotetext{
* Correspondence: ckenyon@itg.be

${ }^{1} \mathrm{HIV} / \mathrm{STI}$ Unit, Institute of Tropical Medicine, Antwerp, Belgium

${ }^{2}$ Division of Infectious Diseases and HIV Medicine, University of Cape Town,

Anzio Road, Observatory, Cape Town 7700, South Africa
}

the right shift. Right shifting could occur via a number of pathways. Firstly, if a subpopulation of $N$. gonorrhoeae acquires AMR this could lead to a bimodal distribution in the MIC with no/little change in the MIC of the original 'wild type' susceptible population (Fig. 1, type A, stage 1) [6]. In a second stage of this process and following further selection pressure, the most susceptible gonococci could become rare. The new 'wild type' gonococci would now constitute a new MIC peak with a right-shifted unimodal distribution (type A, stage 2). This distribution could then develop a new subpopulation with even higher MICs resulting in a new bimodal distribution (Type A, stage 3 etc.). Secondly, the acquisition of AMR could be followed by a zero-sum stretching of the entire MIC distribution where the isolates in the left-hand tail are reduced in direct proportion to the increase of isolates in the right-hand tail (Fig. 1, type B). Thus in a type A stage 1 shift, the

(c) The Author(s). 2019 Open Access This article is distributed under the terms of the Creative Commons Attribution 4.0 International License (http://creativecommons.org/licenses/by/4.0/), which permits unrestricted use, distribution, and reproduction in any medium, provided you give appropriate credit to the original author(s) and the source, provide a link to the Creative Commons license, and indicate if changes were made. The Creative Commons Public Domain Dedication waiver (http://creativecommons.org/publicdomain/zero/1.0/) applies to the data made available in this article, unless otherwise stated. 


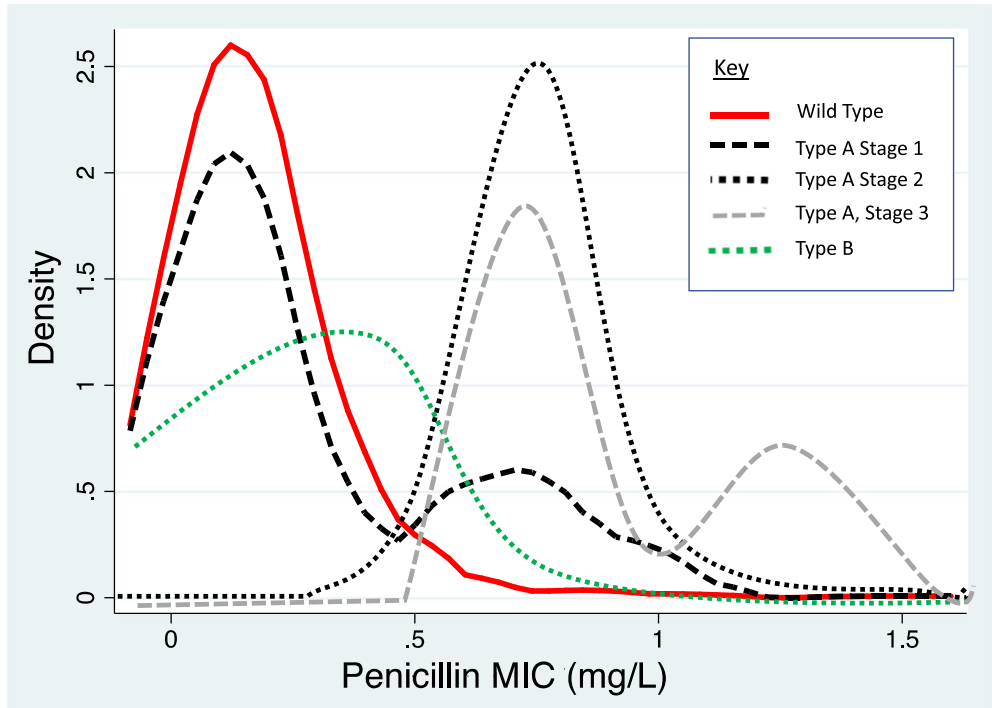

Fig. 1 A schematic representation of how N. gonorrhoeae benzylpenicillin MIC distributions could change over time. A type A shift involves in the first stage, the emergence of a second population of less susceptible isolates (black, long-dashed line). With further selection pressure all the gonococci move into the higher MIC peak resulting in a right-shifted unimodal distribution (type A, stage 2, black, short-dashed line). This distribution could then develop a new subpopulation with higher MICs resulting in a new bimodal distribution (Type A, stage 3; grey, longdashed line). Alternatively, the decrease in susceptibility results from a shift in the whole population (type B shift, green, short-dashed line)

increase in gonococcal AMR leads to a wider MIC distribution. This is then followed, in the second stage, by a reduction in MIC distribution width as most of the highly susceptible isolates are lost through evolution. This process leads to the (close to) elimination of the highly susceptible isolates in type A (stage 2 and 3 ) but not B shifts.

An elimination of highly susceptible strains would likely increase the risk that the loss of susceptibility is irreversible. This may have important consequences for not only gonococcal AMR but also that of commensal bacteria. There is increasing evidence that high antimicrobial exposure increases the risk of AMR in $N$. gonorrohoeae as well as various other Neisseriae and other commensal species [6-9]. If excess antimicrobial consumption results in a type A, stage 2 or 3 trajectory in not only $N$. gonorrhoeae but also in these commensal species then this may be a warning of more wide-ranging adverse effects of antimicrobials on our microbiomes and resistomes [10].

In this review we conducted a non-quantitative historical analysis of how $N$. gonorrhoeae MIC distributions for 3 antimicrobials (benzylpenicillin, ceftriaxone and azithromycin) have changed over time in five countries.

\section{Methods \\ Data}

\section{Historical review}

We selected the five countries, Denmark, Japan, South Africa, the United Kingdom (UK) and the United States (USA) for the study based on the availability and quality of data over a long enough time period to answer our study question. Further selection considerations included trying to ensure a mix of regions or countries with the best quality data from early on in the antibiotic era (Denmark, the UK and the USA). We included Japan since this country has been noted to be among the first where AMR has arisen on a number of occasions [2, 11]. Whilst gonorrhoea transmission disproportionately affects coregroups in these three high income countries, this is not the case in South Africa which was chosen to represent a low to middle income country where most transmission has been in the general heterosexual population [12].

\section{Search strategy and selection criteria}

We conducted a literature review using PUBMED and Google Scholar to find studies reporting minimum inhibitory concentrations (MIC) or 50\% inhibitory concentration (IC50) distributions from 1940 to the present. MeSH terms used in the PubMed search (search date: 10/03/2019) were "Denmark" OR "Japan" OR "South Africa", OR "United States" OR "United Kingdom" OR "England" OR "Scotland" OR "Wales" OR "Ireland”; AND: "gonorrhoea” OR "gonorrhea" OR "Neisseria gonorrhoeae"; filters: publication date from 01/01/1940 until 09/03/2019. Similar search terms were used for the Google Scholar search (search date: 30/ 03/2019). In addition we searched national surveillance websites for AMR reports: Denmark (www.ssi.dk), Japan (www.niid.go.jp), South Africa (http://www.nicd.ac.za), United Kingdom (https://www.gov.uk/government/publications/gonococcal-resistance-to-antimicrobials-surveillanceprogramme-grasp-report) and the United States (https:// 
www.cdc.gov/std/gisp/default.htm). No studies from the Republic of Ireland were included.

\section{Inclusion and exclusion criteria}

Studies were eligible for inclusion if they reported the IC50 or MIC distributions for any of the three antibiotics and the following was true:

The sampling strategy was not overtly biased. Samples needed to either be from all or a non-selected group of patients attending primary care level STI clinics, or based on national AMR surveillance reports. We excluded all studies which reported samples where higher AMR was probable - such as samples which had suspected AMR sent for confirmatory testing.

We selected the studies reporting MIC distributions from isolates at the earliest and latest dates from each country for each antimicrobial. In addition where ever possible we selected a MIC distribution from the midpoint between these two time points. All data are presented as MICs or IC50s. The 1945 benzylpenicillin susceptibility data from the USA are reported in Oxford units of benzylpenicillin per ml. We multiplied this by 1667 to convert Oxford units to mg of benzylpenicillin [13].

Contemporary EUCAST breakpoints were used to define AMR: azithromycin resistance, $>0.5 \mathrm{mg} / \mathrm{L}$; ceftriaxone resistance, $>0.125 \mathrm{mg} / \mathrm{L}$; and benzylpenicillin resistance, > $1 \mathrm{mg} / \mathrm{L}$ (available at: http://www.eucast.org).

\section{Comparisons with EUCAST's wild type MIC distributions}

We also compare the MIC distributions of the three antimicrobials with those from the European Committee on Antimicrobial Susceptibility Testing (EUCAST) "Antimicrobial wild type distributions of microorganisms" collection [14]. EUCAST has used this data to establish epidemiological cut-offs (ECOFFs) for ceftriaxone $(0.032 \mathrm{mg} / \mathrm{L})$ and azithromycin $(1 \mathrm{mg} / \mathrm{L})$, but not for benzylpenicillin $[14,15]$. For these visual comparisons we compare the EUCAST MIC distributions with the earliest country distribution.

\section{Statistical analysis}

All analyses were performed in STATA 13.0 (StataCorp LP, College Station, TX, USA). When exact figures were not reported, IC50/MIC distributions were digitalized using GetData Graph Digitizer 2.26. For graphical representation of MIC distributions, if a study reported the lowest MIC distribution as less than or equal to a particular MIC then this MIC value was used.

\section{Results}

\section{Historical review}

A total of 1203 articles were identified, and 74 articles reviewed (Fig. 2). Twenty-eight publications were selected to provide the MIC distributions. A summary of the study characteristics used to provide these distributions is given in Table 1.

\section{Benzylpenicillin \\ Denmark}

A study by Reyn et al., utilized the same methodology to assess the IC50s of local isolates from 1944 and 1957, in parallel [16]. The IC50 distribution in 1944 was normally distributed with a mode of $0.0144 \mathrm{mg} / \mathrm{L}$. By 1957 the distribution was bimodal with a first peak that was largely unchanged from 1944 and a second peak centered around an IC50 of $0.2 \mathrm{mg} / \mathrm{L}$ (Fig. 3).

We could not find papers published after this date that reported benzylpenicillin IC50 or MIC distributions. A Danish surveillance report from 2001 however reported that $40 \%$ of 334 isolates in 1999 were benzylpenicillin resistant (MIC $>1 \mathrm{mg} / \mathrm{L}$ ) and that this figure was unchanged for 5 years [35]. Euro-GASP does not report MIC distributions per country and thus we were unable to obtain this data from this source.

\section{Japan}

Between 1968 and 2009 the MIC range increased from $0.125-1$ to $0.06-64 \mathrm{mg} / \mathrm{L}[17,18]$. The lowest dilutions tested were 0.125 in 1968 and $0.06 \mathrm{mg} / \mathrm{L}$ in 2009. The modal MIC increased from 0.5 to $1 \mathrm{mg} / \mathrm{L}$ (Fig. 3).

\section{South Africa}

The earliest benzylpenicillin MIC distribution was from 1976 and was not dissimilar to the Denmark bimodal distribution in 1957 with peaks at 0.01 and $0.5 \mathrm{mg} / \mathrm{L}$ (range 0.006 to $1 \mathrm{mg} / \mathrm{L}$ ) [36]. By 2013, the bimodal MIC distribution was retained but right shifted with two peaks at 0.25 and $8 \mathrm{mg} / \mathrm{L}$ [21]. Only $1.6 \%$ of isolates in $2013 \mathrm{had}$ a MIC $\leq 0.06 \mathrm{mg} / \mathrm{L}$. Less than half (38.6\%) of isolates from 2013 had MICs that overlapped with those from 1976 (Fig. 3).

UK

In 1957 the benzylpenicillin MIC was approximately bimodally distributed, with peaks at 0.008 (the modal value) and $0.25 \mathrm{mg} / \mathrm{L}$ [23]. By 2001, the range had increased from $\leq 0.008-0.5$ to $\leq 0.03-8$ with a right shifted modal value of $0.125 \mathrm{mg} / \mathrm{L}$. The lowest dilutions tested were 0.008 in 1957 and $0.03 \mathrm{mg} / \mathrm{L}$ in 2001. The MIC distribution from the midpoint between these two time points was from 1994 and was somewhat right shifted compared to that from 2001. The 2001 data were provided by the UKs' national surveillance Gonococcal resistance to antimicrobials surveillance programme (GRASP, [25]). Unfortunately, GRASP did not report the benzylpenicillin MIC distribution after 2001. 


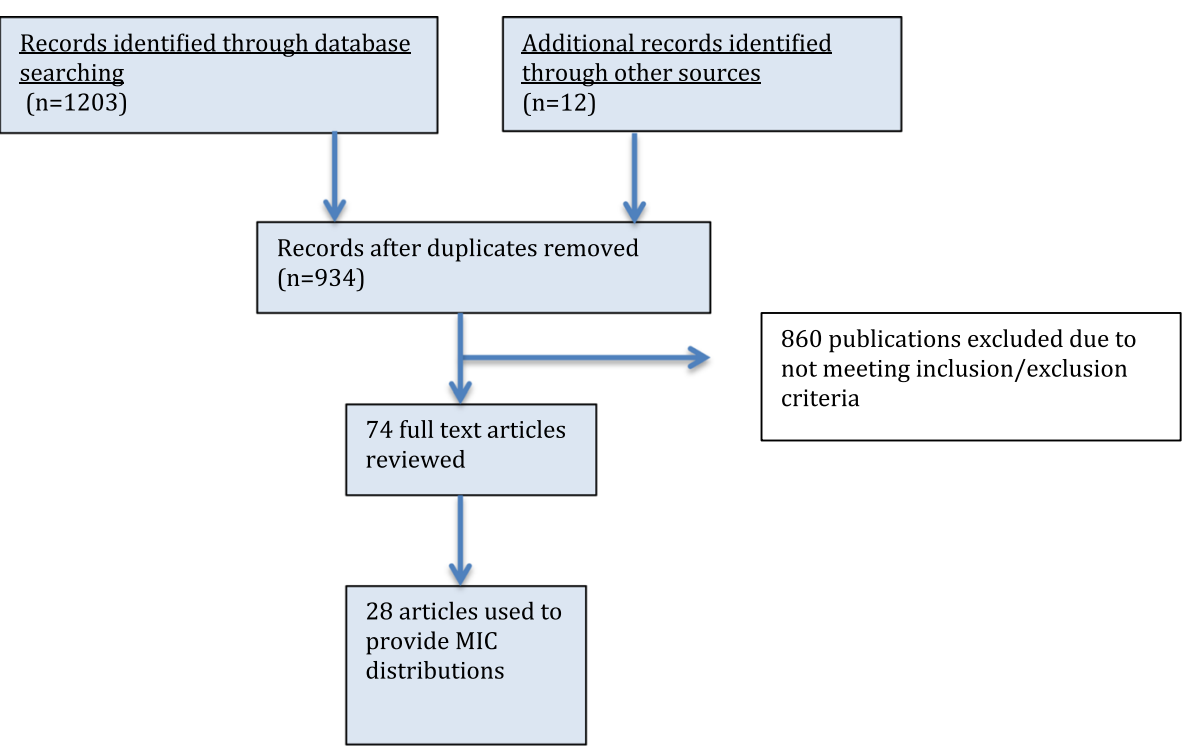

Fig. 2 PRISMA flow chart showing selection of publications from the literature search

\section{USA}

Between 1945 and 1987, the benzylpenicillin MIC distribution shifted from $0.0015-0.12$ to $0.008-8 \mathrm{mg} / \mathrm{L}$ [29, 31]. Only $1.5 \%$ of the 1987 distribution overlapped with the 1945 distribution. The mode increased from 0.006 to $0.125 \mathrm{mg} / \mathrm{L}$. A study from a single laboratory in Boston that used the same MIC methodology (agar dilution) to test susceptibility for gonococcal isolates obtained before 1947 with those from 1949, found an increase in the mode from 0.003 to 0.02 and a right shifting of the distribution from $0.001-0.005$ to $0.003-0.2 \mathrm{mg} / \mathrm{L}$ (Fig. 3) [30].

\section{EUCAST}

The EUCAST wild type penicillin MIC distribution was so right shifted compared to the distribution from 1945 in the USA that there is almost no overlap in their distributions (Fig. 3). The 1945 distribution has a mode of $0.006 \mathrm{mg} / \mathrm{L}$ and a range up to $0.012 \mathrm{mg} / \mathrm{L}$. The corresponding values for the EUCAST distribution are $0.125 \mathrm{mg} / \mathrm{L}$ and $64 \mathrm{mg} / \mathrm{L}$.

\section{Azithromycin \\ Japan}

The azithromycin MIC range increased from 0.016-1 $\mathrm{mg} / \mathrm{L}$ in $1992 / 93$ to $0.015-2 \mathrm{mg} / \mathrm{L}$ in 2013 [3, 20]. The MIC50 increased from 0.125 to $0.25 \mathrm{mg} / \mathrm{L}$ over this time period. In a two-year period, a study from Sendai, Japan, found a slight right shifting of the MIC distribution between 2014 and 2015, with the range increasing from $0.03-1$ to $0.06-16 \mathrm{mg} / \mathrm{L}$ (Fig. 4) [11].

\section{South Africa}

The prevalence of azithromycin resistance increased from 5.4 to $68.0 \%$ between 1999 and 2013 in the same clinic in South Africa [21, 22]. This was associated with a right shift of the entire MIC distribution. The modal value increased from a MIC of 0.12 to $1 \mathrm{mg} / \mathrm{L}$ over this time period. The percent of isolates with a MIC of $\leq 0.5$ $\mathrm{mg} / \mathrm{L}$ decreased from 85 to 3\% (Fig. 4).

\section{UK}

There was a slight increase in the range of azithromycin MICs between 2001 and 2015 and an increase in modal MIC from 0.12 to $0.25 \mathrm{mg} / \mathrm{L}[25,26]$. This was associated with a decrease in the proportion of isolates with low MICs (Fig. 4). The midpoint MIC distribution (2003) revealed a MIC distribution that was intermediate between those of 2001 and 2015 .

\section{USA}

Between 1992 and 2017 there was an increase in the modal azithromycin MIC from 0.06 to $0.25 \mathrm{mg} / \mathrm{L}$ and an increase in the MIC range from $0.03-0.5 \mathrm{mg} / \mathrm{L}$ to $0.03-$ $16 \mathrm{mg} / \mathrm{L}$. The midpoint MIC distribution was from 2013 and was very similar to that of 2017.

\section{EUCAST}

The EUCAST wild type azithromycin MIC distribution was right shifted compared to the distribution from 1992 in the USA (Fig. 4). The modal MIC and MIC range for EUCAST were both one dilution higher.

\section{Ceftriaxone \\ Japan}

Between 1995 and 2005 there was a large increase in the prevalence of isolates with decreased-susceptibility to ceftriaxone (MIC $>0.125 \mathrm{mg} / \mathrm{L})$ from $0 \%$ of 34 isolates 
Table 1 Sources and study methodology of benzylpenicillin, ceftriaxone and azithromycin MIC data used in the study

\begin{tabular}{llll}
\hline Country/ Antimicrobial Sampling period Study design, testing modality and study reference References \\
EUCAST
\end{tabular}

Denmark

Benzylpenicillin 1944 \& 1957

1944: 90 strains from 90 non-selected patients stored as lyophilized cultures

2009-2010

Ceftriaxone

1995-2005

2010-2013 in accordance with CLSI guidelines.

$1995-2005$

Azithromycin 1981 to 1984 and 1992 to 1993

$2014 / 2015$ and tested in 1957 in parallel with and using the same methodology as the 1957 samples as detailed below.

1957: 103 isolates from 96 randomly-selected patients. Benzylpenicillin susceptibility was assessed using the plate dilution and tablet methods. The two methods provided highly concordant results. Results are reported as $50 \%$ inhibitory concentrations for the plate dilution method which provided the most reproducible results. The $50 \%$ inhibitory concentrations were calculated by means of the Karber method.

33 gonococcal isolates from 33 consecutive navy personnel attending a USA navy STI clinic at Yokosuya, Japan, with a diagnosis of urethritis. MICS were determined via agar dilution following CLSI guidelines.

83 isolates from 83 individuals with gonococcal male urethritis presenting to one of 51 facilities in Japan between April 2009 and October 2010 were sent to a central laboratory for benzylpenicillin MIC testing via agar dilution

MICs were determined using an agar dilution method with a GC agar base containing 1\% defined growth supplement. Performed on 690 clinical isolates of N. gonorrhoeae obtained from local STI clinics between 1995 and 2005 in Kanagwa, Japan. containing $1 \%$ defined growth supplement. Performed on 677 clinical isolates of N. gonorrhoeae obtained from the local STI clinic between January 2010 to December 2013 in Fukuoka, Japan.

175 isolates from non-selected men with urethritis presenting to a GUM clinic in Johannesburg between 1976 and 1977. MICs tested via agar diffusion using Oxoid DST agar. syndrome presenting with urethritis and women with vaginal discharge MICs were tested using the agar dilution method. MICs tested via agar diffusion using Oxoid DST agar.

56 isolates from men with urethritis and women with vaginal discharge syndrome presenting to a single GUM clinic in Durban. MIC testing was performed with agar diffusion using Oxoid DST agar. syndrome presenting to two GUM clinics in Durban and Pietermaritzburg. MICs were tested using the agar dilution method. MICs tested via agar diffusion using Oxoid DST agar.

In 1995, 61 isolates and in 199958 isolates from men with urethritis and women with vaginal discharge syndrome presenting to a single GUM clinic in Durban. MIC testing was performed with agar diffusion using Oxoid DST agar. syndrome prom men with urethritis and women with vaginal discharge MICs were tested using the agar dilution method. MICs tested via agar diffusion using Oxoid DST agar.

Taken from a report produced by the Medical Research Council Working Party to Examine the Resistance of Gonococci to Benzylpenicillin. Results for 302 isolates taken in the London area in April to December 1957 are 
Table 1 Sources and study methodology of benzylpenicillin, ceftriaxone and azithromycin MIC data used in the study (Continued)

Country/ Antimicrobial Sampling period Study design, testing modality and study reference References

EUCAST

provided. All samples were tested centrally via the same agar diffusion technique.

$1994 \quad$ Isolates were from 113 consecutive patients attending the GUM clinic at an East London GUM clinic over a one-year period. 66\% were from men and 3.5\% MSM. MICs were determined by agar diffusion.

2001 In the 2001 Gonococcal resistance to antimicrobials surveillance programme (GRASP) report, 2542 isolates from 24 participating GUM clinics had their MICS ascertained via agar diffusion using Oxoid DST agar.

Ceftriaxone 2003, $2008 \quad 2003$ results are taken from Gonococcal resistance to antimicrobials surveillance and 2015 programme (GRASP) Report 2003. In this survey 1977 non-duplicate isolates had MIC testing performed with agar diffusion using Oxoid DST agar. Results for 2008 and 2015 are taken from the 2016 Gonococcal resistance to antimicrobials surveillance programme (GRASP) report [26]. 2008 data: 1276 isolates were tested from 26 GUM clinics in England and Wales. MIC testing performed with agar diffusion using Oxoid DST agar. No further details provided. 2015 data: 2302 isolates from 1699 unique patients from 23 English GUM clinics were tested. $87 \%$ were men and $72 \%$ were MSM. Slight over-representation of MSM and London residents. MIC testing performed with agar diffusion using HiMedia DST agar.

In the 2001 Gonococcal resistance to antimicrobials surveillance programme (GRASP) report, 2542 isolates from 24 participating GUM clinics had their MICs ascertained via agar diffusion using Oxoid DST agar. $71 \%$ were men and $25 \%$ MSM.

20032003 results are taken from Gonococcal resistance to antimicrobials surveillance programme (GRASP) Report 2003. In this survey 1977 non-duplicate isolates had MIC testing performed with agar diffusion using Oxoid DST agar. $72 \%$ were from men and 24\% from MSM. $47.1 \%$ were from London and $52.9 \%$ from outside London. Azithromycin MIC distributions for the whole country were not provided but distributions for both London and non-London populations were provided. These were similar and we use the data for the non-London sample as this was larger.

20152302 isolates from 1699 unique patients from 23 English GUM clinics were tested. $87 \%$ were men and $72 \%$ were MSM. Slight over-representation of MSM and London residents. MIC testing performed with agar diffusion using HiMedia DST agar

USA Benzylpenicillin 1945

Pre-1947 and 1949

104 isolates from a non-selected group of female patients with a diagnosis of gonorrhoea attending a clinic at the University of Texas. MIC were tested via agar dilution on $0.5 \%$ starch agar.

A study from a single laboratory in Boston that used the same MIC methodology (agar dilution) to test susceptibility for gonococcal isolates obtained before 1947 $(n=24)$ with those from $1949(n=52)$. Isolates were from men and women. Heart-infusion agar was used in all assessments.

Benzylpenicillin, 1987/1988, 1992, The typical annual Gonococcal Isolate Surveillance Project (GISP) methodology is azithromycin \& 2013 \& 2017 ceftriaxone as follows: Isolates are collected monthly from up to the first 25 men with $\mathrm{N}$. gonorrhoeae urethritis attending participating STD clinics. Isolates are sent to the regional laboratories for agar dilution antimicrobial susceptibility testing.

GISP 1987 (benzylpenicillin/ceftriaxone): 6204 isolates from men with urethritis from 21 clinics presenting between September 1987 and December 1988. MICs were ascertained at 4 central laboratories using the agar-dilution technique with GC-II Base medium [31].

GISP 1992 (Azithromycin) Susceptibility testing for azithromycin in GISP began in 1992. GISP 1992 tested 5238 gonococcal isolates [32, 33]

GISP 2013: (ceftriaxone/azithromicin): In GISP 2017 STD clinics affiliated with 27 state or city health departments sent 5093 gonococcal isolates to GISP. GISP 2017 (ceftriaxone/azithromicin): In GISP 2017 STD clinics affiliated with 27 state or city health departments contributed 5061 gonococcal isolates to GISP.

EUCAST

Azithromycin -

3727 isolates from 10 collections 
i

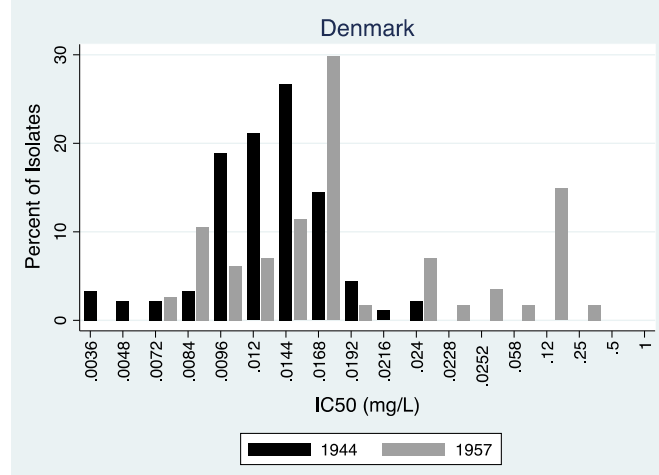

iii

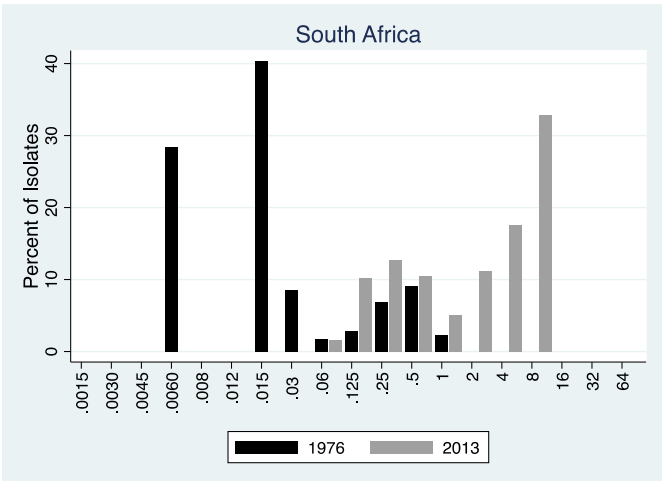

v

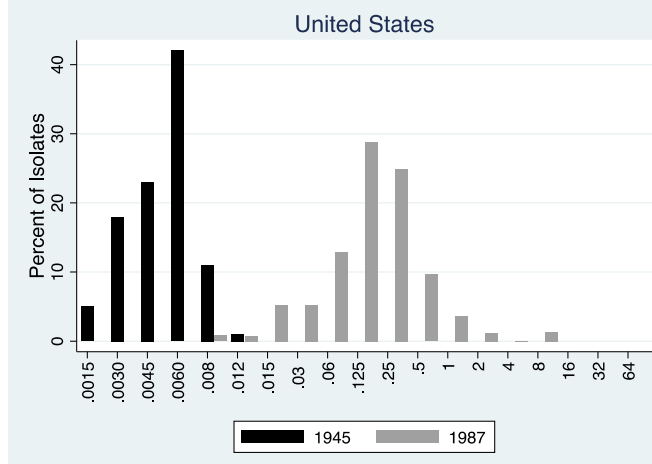

vii

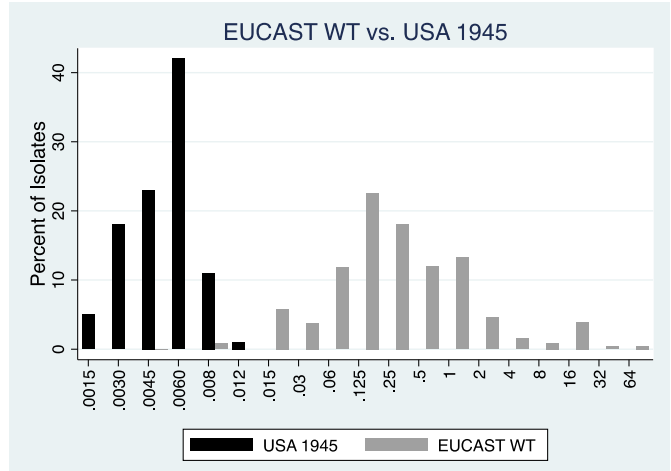

ii

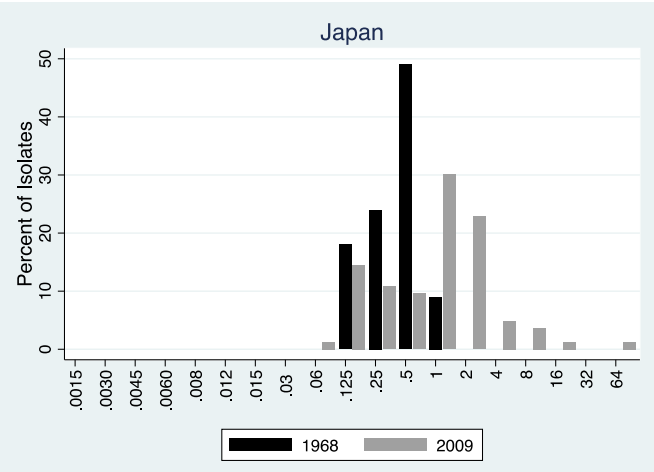

iv

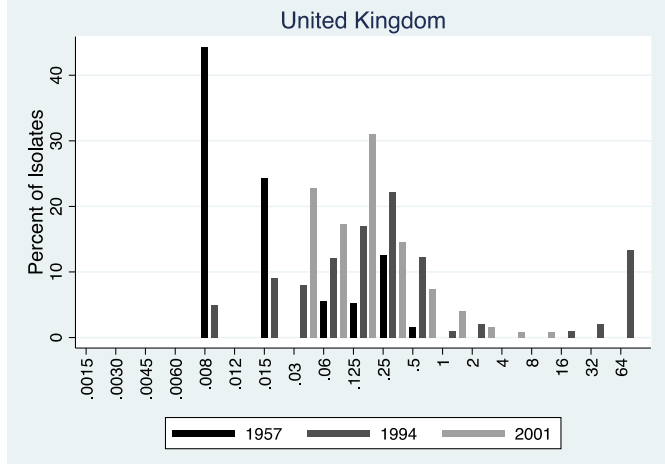

vi

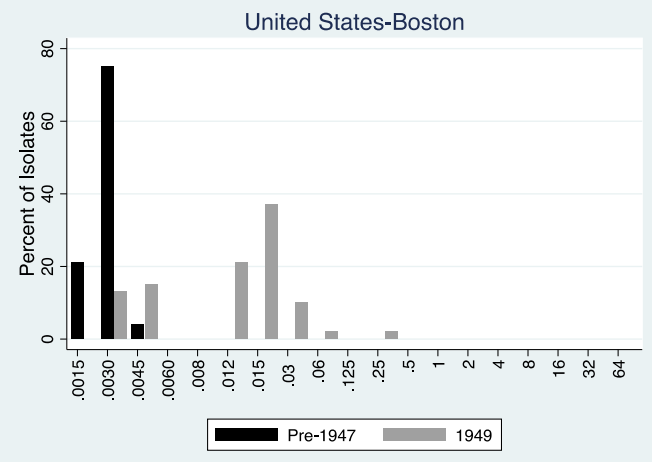

Fig. 3 (See legend on next page.) 
(See figure on previous page.)

Fig. 3 Distribution of Neisseria gonorrhoeae benzylpenicillin 50\% inhibitory concentrations (IC50) distributions for Denmark (i) and minimum inhibitory concentrations (MIC) distributions for Japan (ii), South Africa (iii), United Kingdom (iv), United States (v), Boston (United States) (vi), and EUCAST wild type collection (EUCAST WT) (vii). All MIC values are reported in mg/L. Please note that the $x$-axis between 0.015 and $64 \mathrm{mg} / \mathrm{L}$ uses a doubling scale and below $0.015 \mathrm{mg} / \mathrm{L}$ a less than doubling scale to be able to more accurately represent the distribution of more susceptible strains in the earlier studies

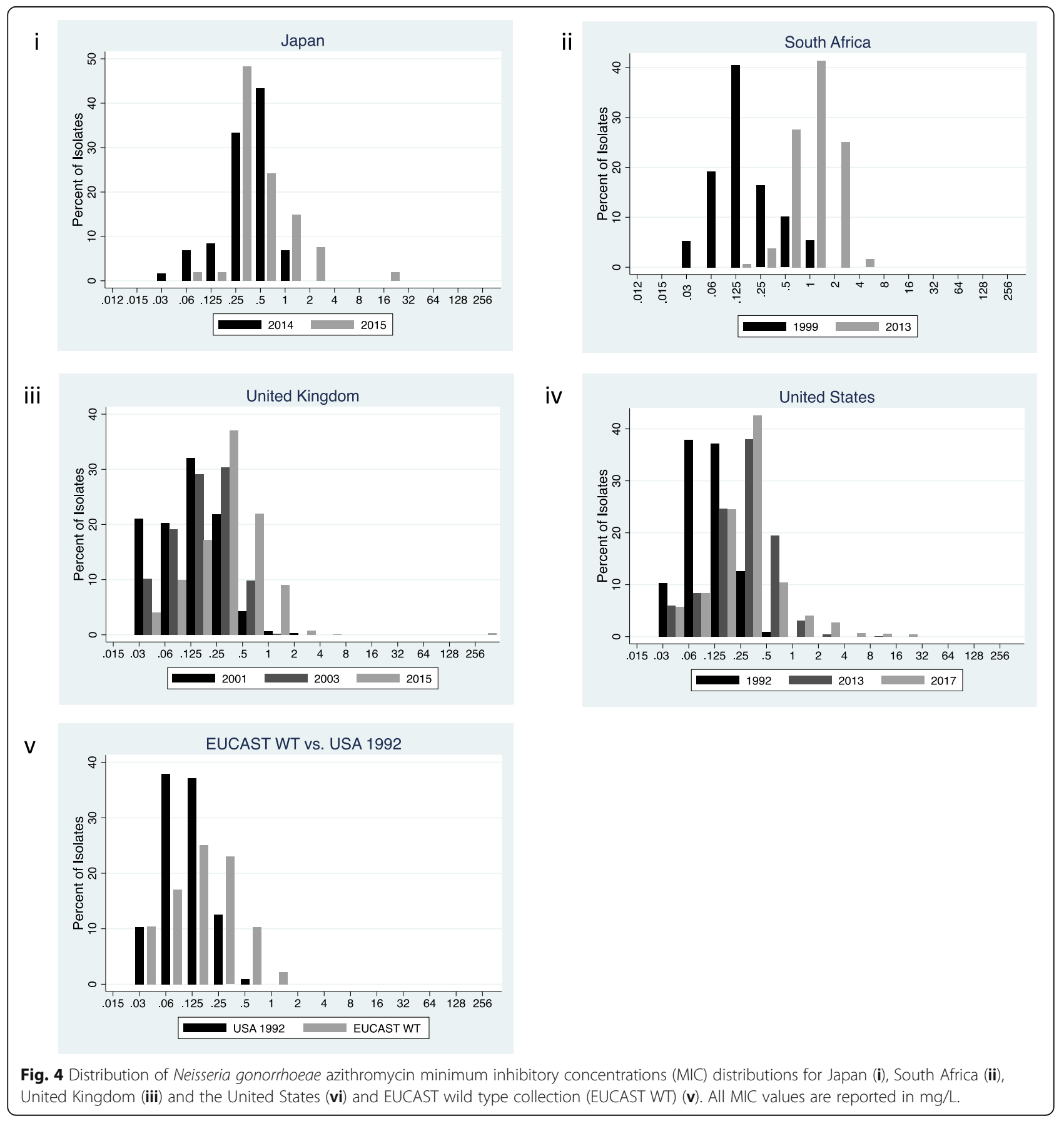


in 1995 to $29.1 \%$ of 55 isolates in 2003 and $14.7 \%$ of 34 isolates in 2005 [19]. A commensurate increase in cefixime resistance $(\mathrm{MIC}>0.125 \mathrm{mg} / \mathrm{L})$ was noted from $2.9 \%$ in 1995 to $47.3 \%$ in 2003 and $29.4 \%$ in 2005, in Kanagawa, Japan [19]. These studies did not report MIC distributions and thus this could not be graphically depicted. An unpublished study from the same last author did however provide MIC distributions from 2001 and 2007 and this demonstrated a large decrease in the proportion with ceftriaxone in the lowest MIC categories tested - from 42 to $0.5 \%$ for $\mathrm{MIC}$ of $<0.03 \mathrm{mg} / \mathrm{L}$ (Fig. 5) [37]. This was associated with commensurate increases in higher MICs without an increase in the MIC range. Between 1993/94 and 2013 there was little movement in the MIC range $(\leq 0.001-0.25$ to $0.001-0.12$, respectively) and MIC50-0.016 and 0.015, respectively [3]. Studies from elsewhere in Japan showed increases in ceftriaxone and cefixime MICs [2].

\section{South Africa}

In $1995,100 \%$ of isolates were in the lowest MIC category tested $(\leq 0.007 \mathrm{mg} / \mathrm{L})$. By 1999 , this had dropped to $53 \%$ [22]. These data were obtained using same methodology testing patients attending the same clinics. By 2013 the proportion with MICs of $\leq 0.007 \mathrm{mg} / \mathrm{L}$ had increased again to $77 \%$ (Fig. 5) [21].

\section{UK}

Between 2003 and 2015, the modal MIC increased from $<0.002$ to $0.008 \mathrm{mg} / \mathrm{L}$ and the highest MIC increased from $0.008 \mathrm{mg} / \mathrm{L}$ to $0.3 \mathrm{mg} / \mathrm{L}$ [26]. This was associated with a reduction of isolates in the lowest MIC category over this time course (76.8 to $4 \%$; Fig. 5). The MIC distribution for 2008 was not substantially different, though a slight right shift may be present.

\section{USA}

The lowest ceftriaxone concentration tested was 0.001 in 1987 and $0.008 \mathrm{mg} / \mathrm{L}$ in 2017 [31, 34]. The distribution of strains with MIC below $0.008 \mathrm{mg} / \mathrm{L}$ can therefore not be compared between these years. In $20170.2 \%$ of isolates had MICs $\geq 0.125 \mathrm{mg} / \mathrm{L}$. This proportion was not reported in 1987, when the highest MIC reported was $\geq 0.06$, but the GISP reports note that this proportion has not changed since 2012 [34]. The MIC distribution in 2013 was similar to that in 2017.

\section{EUCAST}

The EUCAST wild type ceftriaxone MIC distribution was right shifted compared to the distribution from 1987 in the USA (Fig. 5).

\section{Discussion}

Our results provide support for both the bimodal (type A) and the general shift (type B) of MIC distributions over time. The changes in MIC distribution for ceftriaxone fit best with a type B shift where the key change in MIC distribution has been an increase in the prevalence of isolates with higher MICs and a commensurate decrease but not elimination of isolates with low MICs. This is most clearly seen in South Africa where in 1995, 100\% of isolates had a MIC of $\leq 0.007 \mathrm{mg} / \mathrm{L}$, but by 2013 this had been reduced to $77 \%$. In all three countries, where this could be assessed, the gonococci with the lowest MIC assessed were not eradicated over time.

The changes in MIC distribution for benzylpenicillin fit better with a type A shift. In Boston, for example, the pre1947, pre-penicillin isolates are approximately normally distributed around a MIC of $0.003 \mathrm{mg} / \mathrm{L}$. By 1949, a new sub-population of more resistant isolates has emerged centering on a MIC of $0.015 \mathrm{mg} / \mathrm{L}$ (type A, stage 1). By 1987 , the USA distribution is approximately normal once again but now centered around a MIC of $0.125 \mathrm{mg} / \mathrm{L}$ (type A, stage 2). Only $1.5 \%$ of the 1987 isolates had a MIC of $\leq 0.012 \mathrm{mg} / \mathrm{L}$.

Similarly, in Denmark, the introduction of penicillin is followed by a new bimodal distribution (type A, stage 1). The earliest benzylpenicillin MIC distributions from South Africa (1976) and the UK (1957) are from the penicillin period and demonstrate bimodal distributions. The later distributions from these countries are right shifted compared to the earlier period and in the case of South Africa, exhibit a new bimodal distribution including a new population centered on a MIC of $8 \mathrm{mg} / \mathrm{L}$ (type A, stage 3 ). The earliest data from Japan (1968) do not demonstrate a bimodal peak but a unimodal distribution centered around a high MIC of $0.5 \mathrm{mg} / \mathrm{L}$. By 2009, similar to South Africa, bimodality has emerged as a result of the emergence of a new population with MICs around $1 \mathrm{mg} / \mathrm{L}$ (type A, stage 3 ).

The results for azithromycin are more mixed. South Africa's trajectory fits best with type A, stage 2 - the whole MIC distribution is right shifted. In the UK and USA on the other hand, the distribution has been stretched to the right and thus best characterized as type B shifting.

The different trajectories of azithromycin, ceftriaxone and benzylpenicillin may be due to the different length of time they were observed for. This hypothesis is compatible with the fact that benzylpenicillin which has the longest follow-up data available exhibited the most marked changes in MIC distribution. As longer followup data become available for ceftriaxone and azithromycin they may follow a similar trajectory to benzylpenicillin. Formally defining type A and B shifts was beyond the scope of this analysis. We therefore acknowledge that our classification of MICs shifts into these types will remain open to debate until larger datasets are analyzed 


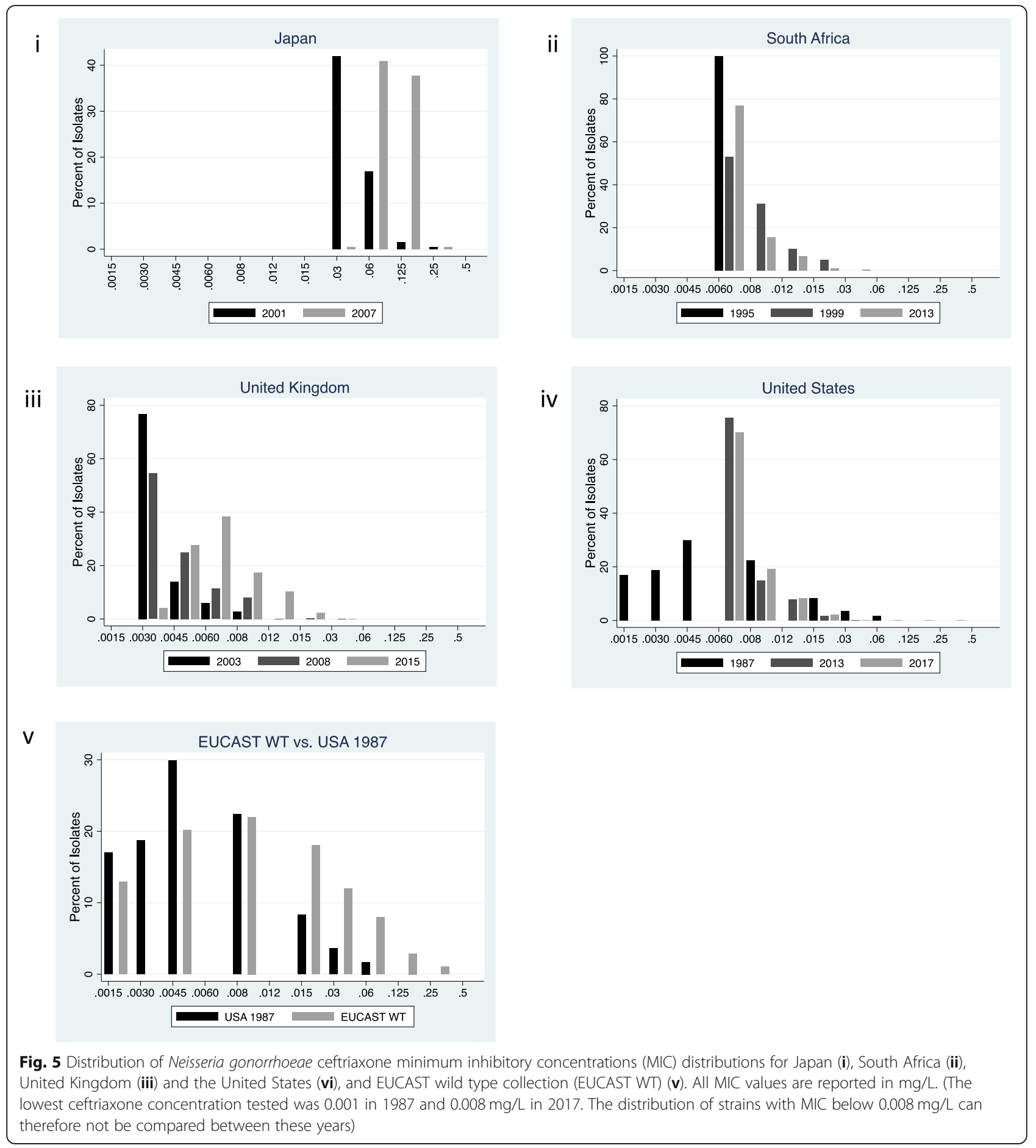

that enables a more formal classification of how MICs change over time per species. A further considerable limitation of these analyses is that changes in MIC distributions between studies may reflect differences in ascertainment of susceptibility. An example of this is a change of supplier of the agar used for susceptibility testing in the UK's GRASP in 2015, which was shown to lead to slight increases in MICs for benzylpenicillin, ceftriaxone and azithromycin [38]. These limitations would not however apply to the benzylpenicillin analyses from Denmark where the isolates from both periods were tested in parallel using the same methodology. Further limitations include the fact that we used the susceptibility data as they were reported and did not assess if publication or selection biases were present or absent. In particular we did not assess if changes in the population 
sampled may have influenced the results. For example, if subpopulations with higher rates of AMR were more likely to be included in the later dates this may lead to a right shifting of MIC distributions. The potential for this bias is particularly relevant in three of our comparisons: 1. Benzylpenicillin, Japan 1968 versus 2009-2010. In 2009 the samples were form men attending an STI clinic whereas the samples from 1968 were from navy personnel who may have been more likely to have obtained their infections from sex workers who have been shown to have more resistant strains in some studies [39], 2. Benzylpenicillin, the USA 1945-1949 versus 1987-onwards. The 1940s isolates were from men and women versus the post 1986 isolates which were all from men. 3. Azithromycin, the UK, 2001 versus 2015. The proportion of samples that were from MSM was considerably higher in 2015 which may been responsible for the right shifting of the MIC distribution [39, 40]. We were also unable to assess if unreported quality issues may have affected the accuracy of the data. Other limitations include that our literature search may have missed published reports, particularly in the early period when the indexing of articles was suboptimal. A further problem was that our analysis was limited to three antimicrobials in four countries, which limits generalizability. Finally, our analysis is descriptive and not quantitative.

Our findings from the pre-penicillin period are however congruent with those from 16 other studies which found that gonococci in this period were highly susceptible to penicillin (reviewed in [16]). Likewise, our findings of a bimodal penicillin MIC distribution emerging soon after significant penicillin exposure are similar to those in multiple populations around the world [41-43]. There is also evidence from other populations not included in our review of the emergence of a second bimodal peak in the penicillin MIC distribution in more recent decades [24, 44]. These studies thus provide some support for our finding that reductions in susceptibility to penicillin, at least in certain populations, are best characterized by type A shifts that evolve through up to 3 stages. By the third stage, benzylpenicillin MIC distributions such as those from Japan and South Africa were so right-shifted that no or very few isolates have MICs $\leq 0.03 \mathrm{mg} / \mathrm{L}$. Their distribution no longer overlaps with that of the pre-penicillin population. How can we explain this?

\section{What mechanisms underpin the decline/extinction of the lowest MIC populations?}

We propose that 3 mechanisms may be relevant: 1) The zero-sum nature of changes in distributions means that an increase in the proportion with high MICs necessarily entails a commensurate decrease in the proportion with lower MICs; 2) Sustained antimicrobial exposure may have led to a selective sweep of $N$. gonorrhoeae whereby highly sensitive strains were eliminated from the gene pool/local pangenome; 3) Sustained antimicrobial pressure may have selected for antimicrobial resistance in commensal bacteria, including commensal Neisseria spp. If this change is profound enough and there is sufficient DNA exchange with these commensals then this could result in the decline or elimination of low MIC strains in both commensal and pathogenic species.

The first mechanism is able to explain a decline but not the elimination of low MIC isolates. Support for the second mechanism comes from studies that have found associations between the intensity of antimicrobial consumption in the general population and homologous gonococcal AMR $[6,9]$. Furthermore, studies have found that particular gonococcal genogroups can emerge and disseminate rapidly displacing other genogroups. For example, genogroup 1407, which is strongly associated with reduced cephalosporin susceptibility, emerged explosively in Europe around 2007 and rapidly displaced other more susceptible genogroups [45]. In support of the third mechanism, recent receipt of ciprofloxacin, ceftriaxone or cefixime has been established as an independent risk factor for homologous AMR in oropharyngeal commensal Neisseria [8]. The relative contributions of mechanisms 2 and 3 is likely to vary between different classes of antimicrobials. For example, horizontal gene transfer of resistance conferring genes from commensal Neisseria has been shown to have played an important role in cephalosporin and macrolide resistance but not for fluoroquinolone resistance [46].

\section{Conclusions}

The major relevance of this study is showing the utility of longer-term analyses of MIC distribution. Contemporary analyses of gonococcal AMR are frequently limited to the last decade or so $[6,9,47]$. These analyses will likely miss changes in MIC distribution that occur slowly over longer periods. In other fields (mainly environmental conservation) this short-term focus has led to what has been termed the shifting baseline syndrome, where in the absence of sufficient knowledge of historical conditions, members of each new generation accept the situation in which they are raised as normal [48]. An important consequence of this syndrome is that it increases an individual's tolerance of man-made environmental damage.

It is important to remember that the emergence of AMR in N. gonorrhoeae is not inevitable. Populations in countries such as the Netherlands with low rates of consumption of macrolides, quinolones and cephalosporins have correspondingly lower rates of gonococcal AMR than high consumption countries [6]. The treatment of choice for gonorrhoea in the rural part of the Northern Territories, Australia, remains penicillin, because the 
prevalence of penicillin resistance here is still less than $1 \%$ [49].

These findings indicate that it is possible to contain the emergence of gonococcal AMR.

The fact that a unimodal distribution can re-emerge in stage 2 of a type A shift may compound the shifting baseline problem. Observers looking at a stage 2 MIC distribution in isolation may conclude that this represents a wild type distribution. Indeed, contemporary datasets are favored in the construction of epidemiological cutoffs (ECOFFs) for MICs [15]. If one analyzes EUCAST's 'Antimicrobial wild type distributions of microorganisms' gonococcal dataset with the EUCAST recommended software "ECOFFinder", this generates an ECOFF for benzylpenicillin of $8 \mathrm{mg} / \mathrm{L}$ [15]. Gonococcal isolates with MICs of over 1 have a high likelihood of non-response to benzylpenicillin therapy [42] which illustrates why it is not meaningful to try to find penicillin ECOFFs for contemporary gonococcal populations that have experienced right shifting of their MICs. Evaluating ECOFFs from the prepenicillin populations would however be more appropriate. Following our historical methodology, the EUCAST ECOFF MIC distribution would be characterized as having undergone a type A, stage 3 shift. Likely because more recent datasets are favored in the EUCAST dataset, the older MIC distributions, such as those from 1945 (Fig. 3), are not included in this distribution.

Further studies could analyze what mechanisms underpin the elimination of highly susceptible strains, what the correlates are of populations that have undergone a stage 3 as opposed to a stage 1 or 2, type A shift in penicillin/other antimicrobial AMR? Can this all be explained by differential antimicrobial exposure? If so are there thresholds of consumption that populations should aim to not exceed [10]? Which gonococcal genotypes/genogroups have been driven extinct in populations with stage 2 and 3 shifts? Is this mirrored by a similar extinction in susceptible strains in commensal species? Finally, it may be useful to investigate if this process has played a role in the reduction in diversity of the gut microbiome in Western populations $[50,51]$.

\section{Abbreviations \\ AMR: Antimicrobial resistance; GISP: Gonococcal isolate surveillance project; GRASP: Gonococcal resistance to antimicrobials surveillance programme; GUM: Genitourinary medicine clinic; MSM: Men who have sex with men; STD: Sexually transmitted diseases; UK: United Kingdom; USA: United States of America}

\section{Acknowledgements}

$\mathrm{Nil}$

\section{Authors' contributions}

CK was responsible for conceptualizing the study and performing the literature reviews, the data analyses and writing the first draft. CK, JL, CVD and DVDB were responsible for data interpretation, editing the first draft and approving the final manuscript.
Funding

No specific funding was received for this work.

Availability of data and materials

The data we used are available from the authors on request.

Ethics approval and consent to participate

Not applicable.

\section{Consent for publication}

Not applicable.

\section{Competing interests}

The authors declare that they have no competing interests.

Received: 20 August 2019 Accepted: 18 December 2019

Published online: 27 December 2019

\section{References}

1. Unemo M, Shafer WM. Antimicrobial resistance in Neisseria gonorrhoeae in the 21st century: past, evolution, and future. Clin Microbiol Rev. 2014;27(3): 587-613.

2. Ito M, Yasuda M, Yokoi S, Ito S, Takahashi Y, Ishihara S, Maeda S, Deguchi T. Remarkable increase in Central Japan in 2001-2002 of Neisseria gonorrhoeae isolates with decreased susceptibility to penicillin, tetracycline, oral cephalosporins, and fluoroquinolones. Antimicrob Agents Chemother. 2004;48(8):3185-7.

3. Tanaka M, Furuya R, Irie S, Kanayama A, Kobayashi I. High prevalence of azithromycin-resistant Neisseria gonorrhoeae isolates with a multidrug resistance phenotype in Fukuoka. Japan Sex Transm Dis. 2015;42(6):337-41.

4. Jacobs MR: Mechanisms of Resistance in Haemophilus influenzae and Moraxella catarrhalis. In: Antimicrob Drug Resist. edn.: Springer; 2017: 867-888.

5. Ison CA, Dillon J-AR, Tapsall JW. The epidemiology of global antibiotic resistance among Neisseria gonorrhoeae and Haemophilus ducreyi. Lancet. 1998;351:S8-S11.

6. Kenyon C, Buyze J, Spiteri G, Cole MJ, Unemo M: Antimicrobial consumption is associated with decreased antimicrobial susceptibility of Neisseria gonorrhoeae in 24 European countries: an ecological analysis. JID 2019, Apr 8. pii: jiz153. doi: https://doi.org/10.1093/infdis/jiz153. [Epub ahead of print].

7. Tedijanto C, Olesen SW, Grad YH, Lipsitch M. Estimating the proportion of bystander selection for antibiotic resistance among potentially pathogenic bacterial flora. Proc Natl Acad Sci U S A. 2018;115(51):E11988-95.

8. Dong HV, Pham LQ, Nguyen HT, Nguyen MXB, Nguyen TV, May F, Le GM, Klausner JD. Decreased Cephalosporin Susceptibility of Oropharyngeal Neisseria Species in Antibiotic-Using Men-who-have-sex-with-men of Hanoi, Vietnam. Clin Infect Dis. 2019.

9. Kenyon C, Buyze J, Wi T. Antimicrobial consumption and susceptibility of Neisseria gonorrhoeae: a global ecological analysis. Front Med. 2018;5:329.

10. Austin DJ, Kakehashi M, Anderson RM. The transmission dynamics of antibiotic-resistant bacteria: the relationship between resistance in commensal organisms and antibiotic consumption. P Roy Soc B-Biol Sci. 1997;264(1388):1629-38.

11. Yasuda M, Ito S, Hatazaki K, Deguchi T. Remarkable increase of Neisseria gonorrhoeae with decreased susceptibility of azithromycin and increase in the failure of azithromycin therapy in male gonococcal urethritis in Sendai in 2015. J Infect Chemother. 2016;22(12):841-3.

12. Johnson $L$, Bradshaw D, Dorrington R. South African comparative risk assessment collaborating $\mathrm{G}$ : the burden of disease attributable to sexually transmitted infections in South Africa in 2000. S Afr Med J. 2007;97(8 Pt 2):658-62.

13. Petri W: Penicillins, cephalosporins, and other $\beta$-lactam antibiotics. Goodman and Gilman's The Pharmacological Basis of Therapeutics 12th Ed McGraw-Hill, New York 2011:1477-1504.

14. Antimicrobial wild type distributions of microorganisms [https://mic. eucast.org/Eucast2/SearchController/search.jsp?action= performSearch\&Beginlndex=0\&Micdif=mic\&Numberlndex=50\&Antib=-1 \&Specium=169]. Accessed 14/04/2019.

15. Turnidge J, Kahlmeter G, Kronvall G. Statistical characterisation of bacterial wild-type MIC value distributions and the determination of epidemiological cut-off values. Clin Microbiol Infect. 2006;12(5):418-25. 
16. Reyn A, Korner B, Bentzon MW. Effects of penicillin, streptomycin, and tetracycline on N-Gonorrhoeae isolated in 1944 and in 1957. Brit J Vener Dis. 1958;34(4):227-39.

17. Keys TF, Halverson CW, Clarke EJ. Single-dose treatment of gonorrhea with selected antibiotic agents. Jama. 1969;210(5):857-61.

18. Hamasuna R, Yasuda M, Ishikawa K, Uehara S, Takahashi S, Hayami H, Yamamoto S, Matsumoto T, Minamitani S, Watanabe A. Nationwide surveillance of the antimicrobial susceptibility of Neisseria gonorrhoeae from male urethritis in Japan. J Infect Chemother. 2013;19(4):571-8.

19. Shimuta K, Watanabe $Y$, Nakayama S, Morita-Ishihara T, Kuroki T, Unemo M, Ohnishi M. Emergence and evolution of internationally disseminated cephalosporin-resistant Neisseria gonorrhoeae clones from 1995 to 2005 in Japan. BMC Infect Dis. 2015;15:378.

20. Tanaka M, Matsumoto T, Kobayashi I, Uchino U, Kumazawa J. Emergence of in vitro resistance to fluoroquinolones in Neisseria gonorrhoeae isolated in Japan. Antimicrob Agents Chemother. 1995;39(10):2367-70.

21. Rambaran S, Naidoo K, Dookie N, Moodley P, Sturm AW. Resistance profile of Neisseria gonorrhoeae in KwaZulu-Natal, South Africa questioning the effect of the currently advocated dual therapy. Sex Transm Dis. 2019;46(4):266-70.

22. Moodley P, Pillay C, Goga R, Kharsany AB, Sturm AW. Evolution in the trends of antimicrobial resistance in Neisseria gonorrhoeae isolated in Durban over a 5 year period: impact of the introduction of syndromic management. J Antimicrob Chemother. 2001;48(6):853-9.

23. Fairbrother RW. Resistance of gonococci to penicillin. Lancet. 1961; 278(7196):226-30.

24. Lewis DA, Ison CA, Livermore DM, Chen HY, Hooi AY, Wisdom AR. A oneyear survey of Neisseria gonorrhoeae isolated from patients attending an East London genitourinary medicine clinic: antibiotic susceptibility patterns and patients' characteristics. Genitourin Med. 1995;71(1):13-7.

25. Public Health England: Gonococcal resistance to antimicrobials surveillance programme. Annual Report, Year 2001. Public Health England: London 2001.

26. Public Health England. Surveillance of antimicrobial resistance in Neisseria gonorrhoeae: Key findings from the 'Gonococcal resistance to antimicrobials surveillance programme' (GRASP) and related surveillance data. Public Health England: London. 2016.

27. Public Health England: GRASP 2008 Report: Trends in Antimicrobial Resistant Gonorrhoea: Public Health England; 2008.

28. Public Health England: Gonococcal resistance to antimicrobials surveillance programme. Annual Report, Year 2003. Public Health England: London 2003.

29. Lankford C. The in vitro tolerance of the gonococcus for penicillin. Am J Syph. 1945;29(1):56-63.

30. Del Love B, Finland M. Susceptibility of Neisseria gonorrhoeae to eleven antibiotics and sulfadiazine. Comparison of susceptibility of recently isolated strains with results obtained in previous years in the same laboratory. Arch Intern Med. 1955;95(1):66-73.

31. Schwarcz SK, Zenilman JM, Schnell D, Knapp JS, Hook EW, Thompson S, Judson FN, Holmes KK. National surveillance of antimicrobial resistance in Neisseria gonorrhoeae. Jama. 1990;264(11):1413-7.

32. Centers for Disease Control and Prevention: Sexually Transmitted Disease Surveillance 1992: Gonococcal isolate surveillance project (GISP) supplement and profiles. Atlanta: US Dep Health Hum Serv 1994.

33. Centers for Disease Control Prevention. Gonococcal Isolate Surveillance Project (GISP) annual report-2002. Atlanta: CDC. 2005;3.

34. Centers for Disease Control and Prevention: Sexually Transmitted Disease Surveillance 2017: Gonococcal isolate surveillance project (GISP) supplement and profiles. Atlanta: US Dep Health Human Serv 2019.

35. Hoffmann S. The laboratory surveillance system of chlamydia trachomatis and Neisseria gonorrhoeae infections in Denmark. Euro Surveill. 2001;6(5):86-90.

36. Robins-Browne RM, Gaillard MC, Koornhof HJ, Schneider J. Antibiotic susceptibility of Neisseria gonorrhoeae isolated in Johannesburg. S Afr Med J. 1978:54(15):601-3

37. Duncan ME. Characterization of mechanisms of antibiotic resistance in Neisseria gonorrhoeae. Geneva: The University of North Carolina at Chapel Hill; 2012.

38. Public Health England. Surveillance of antimicrobial resistance in Neisseria gonorrhoeae in England and Wales: Key findings from the Gonococcal Resistance to Antimicrobials Surveillance Programme (GRASP). PHE: Wellington House. 2016

39. Lewis DA. The role of core groups in the emergence and dissemination of antimicrobial-resistant N gonorrhoeae. Sex Transm Infect. 2013;89(Suppl 4):iv47-51.
40. Kirkcaldy RD, Zaidi A, Hook EW, Holmes KK, Soge O, del Rio C, Hall G, Papp J, Bolan G, Weinstock HS. Neisseria gonorrhoeae Antimicrobial Resistance Among Men Who Have Sex With Men and Men Who Have Sex Exclusively With Women: The Gonococcal Isolate Surveillance Project. Ann Intern Med. 2005-2010;2013, 158(5).

41. Lomholt G, Berg O. The gonorrhea situation in South Greenland in the summer 1964. Br J Vener Dls. 1966;42(2):1-7.

42. Organization WH. Neisseria gonorrhoeae and gonococcal infections: Report of a WHO Scientific Group: WHO; 1978.

43. Willcox R. A survey of problems in the antibiotic treatment of gonorrhoea. With special reference to South-East Asia. Brit J Vener Dis. 1970;46(3):217.

44. Li S, Su X-H, Le W-J, Jiang F-X, Wang B-X, Rice PA. Antimicrobial susceptibility of Neisseria gonorrhoeae isolates from symptomatic men attending the Nanjing sexually transmitted diseases clinic (2011-2012): genetic characteristics of isolates with reduced sensitivity to ceftriaxone. BMC Infect Dis. 2014;14(1):622.

45. Harris SR, Cole MJ, Spiteri G, Sanchez-Buso L, Golparian D, Jacobsson S, Goater R, Abudahab K, Yeats CA, Bercot B, et al. Public health surveillance of multidrug-resistant clones of Neisseria gonorrhoeae in Europe: a genomic survey. Lancet Infect Dis. 2018;18(7):758-68.

46. Wadsworth CB, Arnold BJ, Sater MRA, Grad YH. Azithromycin Resistance through Interspecific Acquisition of an Epistasis-Dependent Efflux Pump Component and Transcriptional Regulator in Neisseria gonorrhoeae. Mbio. 2018;9(4).

47. Wi T, Lahra MM, Ndowa F, Bala M, Dillon JR, Ramon-Pardo P, Eremin SR, Bolan G, Unemo M. Antimicrobial resistance in Neisseria gonorrhoeae: global surveillance and a call for international collaborative action. PLoS Med. 2017;14(7):e1002344.

48. Soga M, Gaston KJ. Shifting baseline syndrome: causes, consequences, and implications. Front Ecol Environ. 2018;16(4):222-30.

49. Lahra MM. Australian Gonococcal surveillance: Australian Gonococcal surveillance Programme annual report, 2013. Commun Dis Intell. 2015;39(1): E137-45.

50. Smits SA, Leach J, Sonnenburg ED, Gonzalez CG, Lichtman JS, Reid G, Knight R, Manjurano A, Changalucha J, Elias JE, et al. Seasonal cycling in the gut microbiome of the Hadza hunter-gatherers of Tanzania. Science. 2017; 357(6353):802-6.

51. Schnorr SL, Candela M, Rampelli S, Centanni M, Consolandi C, Basaglia G, Turroni S, Biagi E, Peano C, Severgnini M, et al. Gut microbiome of the Hadza hunter-gatherers. Nat Commun. 2014;5:3654.

\section{Publisher's Note}

Springer Nature remains neutral with regard to jurisdictional claims in published maps and institutional affiliations.

Ready to submit your research? Choose BMC and benefit from:

- fast, convenient online submission

- thorough peer review by experienced researchers in your field

- rapid publication on acceptance

- support for research data, including large and complex data types

- gold Open Access which fosters wider collaboration and increased citations

- maximum visibility for your research: over $100 \mathrm{M}$ website views per year

At $\mathrm{BMC}$, research is always in progress.

Learn more biomedcentral.com/submission 